in this investigation can most certainly be ascribed to absorbed water. Hoffman and Scott $t^{4}$ have also obtained data indicating that water is responsible for a lowtemperature dielectric loss in PMMA. The fact that absorbed water can also strongly influence certain mechanical properties of PMMA has been noted by McLoughlin and Tobolsky. 5 They studied the dependence of stress relaxation upon moisture content at elevated temperatures. Thus the possibility that small amounts of absorbed water may be responsible for the occasional reports of the $\gamma$ transition in PMMA seems to be a plausible hypothesis.

* Supported in part by the Office of Naval Research.

$\dagger$ United States Rubber Company Predoctoral Fellow.

1 Deutsch, Hoff, and Reddish, J. Polymer Sci. 13, 565 (1954).

2 D. J. Mead and R. M. Fuoss, J. Am. Chem. Soc. 64, 2389 (1942).

3 "Water Absorption in Plexiglas" a report issued by Rohm and Haas Company, January, 1944.

4 Private communication by J. D. Hoffman.

${ }^{5}$ J. R. McLoughlin and A. V. Tobolsky, J. Colloid Sci. 7, 555 (1952).

\section{Orientation in Polyethylene Terephthalate Film}

C. Y. LIANG* aND S. KRTMM

Harrison M. Randall Laboralory of Physics, University of Michigan, Ann Arbor, Michigan

(Received February 14, 1957)

$\mathrm{T}$ HE benzene ring orientation in singly and doubly stretched polyethylene terephthalate films has been determined by means of the effect of specimen tilting on the intensities of infrared bands. Radiation with electric vector polarized perpendicular to the spectrometer slit was used. The specimen was rotated about the vertical axis parallel to the slit, covering the slit at all times. This arrangement has the advantage that differential reflection losses on tilting will be small.

When the film is tilted through an angle $\theta(0 \leqslant \theta<\pi / 2)$ from the position of normal incidence, the intensity of an absorption band $A_{\theta}$ is related to the dipole moment change $\mathbf{M}$ by

$$
A_{\theta}=c N V_{\theta}|\mathbf{M}|^{2} \cos ^{2} \phi
$$

where $c$ is a constant, $N$ the number of dipoles per unit volume, $\phi$ the angle between $\mathbf{M}$ and the electric vector, and $V_{\theta}$ the volume of sample in the absorption path. Since $V_{\theta}$ is proportional to $1 / \cos \theta$,

$$
A_{\theta}=k|\mathbf{M}|^{2} \frac{\cos ^{2} \phi}{\cos \theta},
$$

where $k$ is a constant.

For:

(a) Dipoles randomly oriented: the average value of $\cos ^{2} \phi$ is independent of $\theta$, and $A_{\theta}$ is proportional to $1 / \cos \theta$.

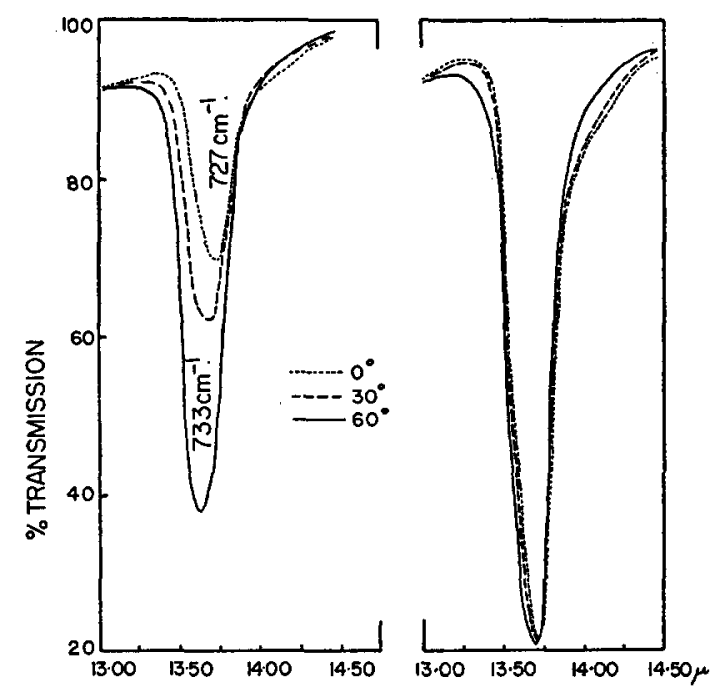

(a)

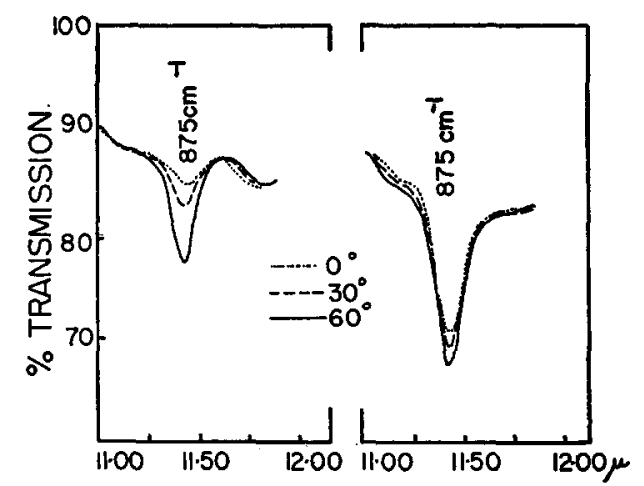

(b)

Frg. 1. Effect of tilting on (a) $730 \mathrm{~cm}^{-1}$ and (b) $875 \mathrm{~cm}^{-1}$ bands of singly stretched polyethylene terephthalate film.

(b) Dipoles normal to film surface: $\cos \phi=\sin \theta$, and $A_{\theta}=k|\mathbf{M}|^{2} \sin ^{2} \theta / \cos \theta$.

(c) Dipoles parallel to film surface and parallel to electric vector at normal incidence: $\cos \phi=\cos \theta$, and $A_{\theta}=k|\mathbf{M}|^{2} \cos \theta$.

(d) Dipoles parallel to film surface and perpendicular to electric vector at normal incidence: $\phi=\pi / 2$, and $A_{\theta}=\mathbf{0}$.

(e) Dipole orientations equally distributed between (b) and (c): this case is similar to (a).

(f) Dipole orientations equally distributed between (b) and (d): $A_{\theta}=k|\mathbf{M}|^{2} \sin ^{2} \theta / \cos \theta$.

(g) Dipole orientations equally distributed between (c) and (d): $A_{\theta}=k|\mathbf{M}|^{2} \cos \theta$.

We consider first the case of a specimen oriented by stretching along one direction. Bands at 730 and 875 $\mathrm{cm}^{-1}$ increase in intensity upon tilting, as is shown in the left-hand portions of Figs. 1(a) and 1(b) (corresponding to the direction of stretch being initially parallel to the electric vector). Preliminary polarized spectra of this sample indicated that the chains are oriented in the 
direction of stretch. The band at $875 \mathrm{~cm}^{-1}$ arises from a $\mathrm{CH}$ out-of-plane bending mode of the benzene ring, while the band at $730 \mathrm{~cm}^{-1}$ most probably has a similar origin. The above observations therefore indicate that the plane of the benzene ring is essentially parallel to the stretching direction. That the orientation of these planes about the stretching direction is otherwise random is shown by tilting a specimen for which the stretching direction was initially perpendicular to the electric vector [shown in the right-hand portions of
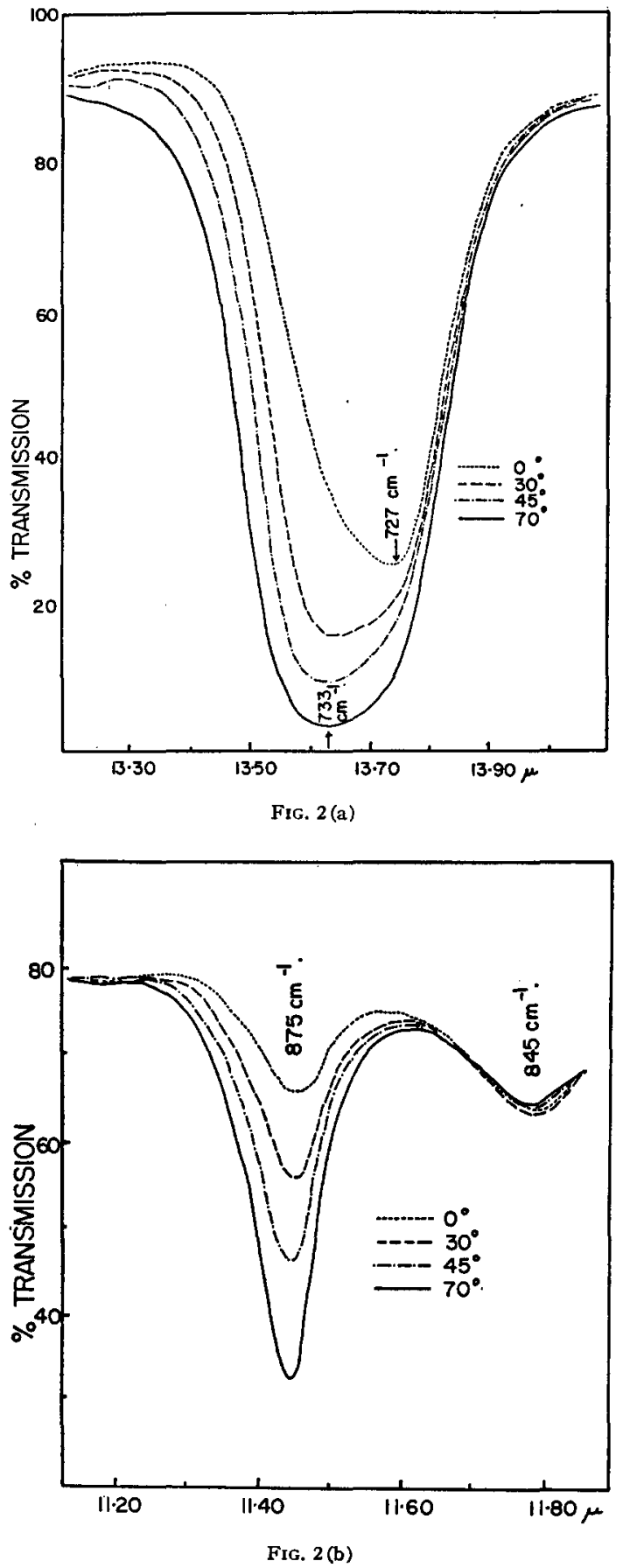

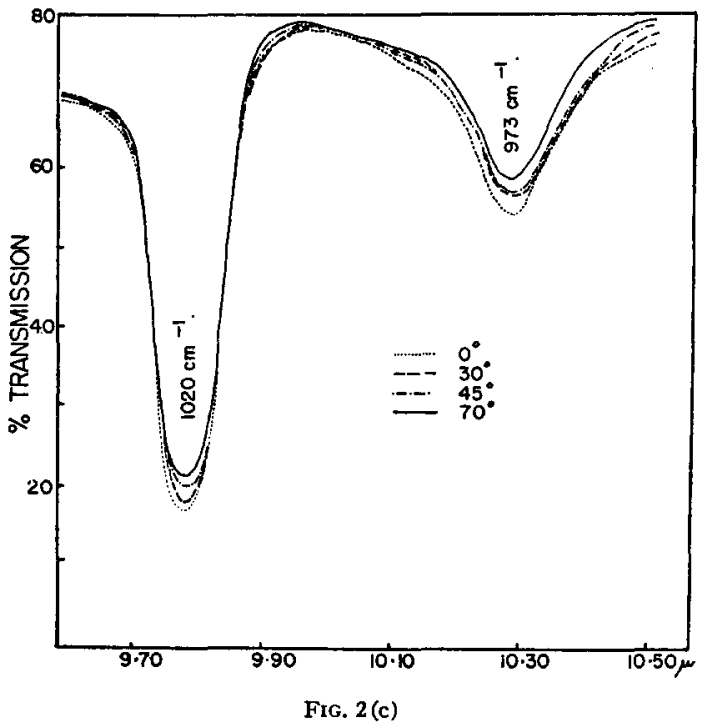

FIG. 2. Effect of tilting on (a) $730 \mathrm{~cm}^{-1}$, (b) $875 \mathrm{~cm}^{-1}$, and (c) $1020 \mathrm{~cm}^{-1}$ bands of doubly stretched $(3 \times 3)$ polyethylene terephthalate film.

Figs. 1(a) and 1(b)]. If the benzene rings were oriented only with their planes parallel to the film surface, the intensity variation would be similar to that previously observed. The relatively smaller increase is consistent with the expected $1 / \cos \theta$ variation as compared with the $\sin ^{2} \theta / \cos \theta$ variation.

The second case is that of a specimen stretched $3 \times$ along one direction and then $3 \times$ perpendicular to this direction. Preliminary polarization studies indicate the absence of any chain orientation in the film. The results of tilting are shown in Figs. 2(a), 2(b), and 2(c), a stretching direction having been initially set parallel to the electric vector. The intensities of the 730 and 875 $\mathrm{cm}^{-1}$ bands again increase on tilting, but the intensity of a band at $1020 \mathrm{~cm}^{-1}$ decreases. This latter band arises from a $\mathrm{CH}$ in-plane bending vibration of the benzene ring. The behavior of both band types thus indicates that the benzene rings are oriented with their planes essentially parallel to the film surface.

* Present address: American Viscose Corporation, Marcus Hook, Pennsylvania.

\section{Photoisomerization Equilibria in Azodyes}

ERnst Fischer and Yaet Frei

Photochemical Laboratory, The Weizmann Institute of Science, Rehovoth, Israel

(Received March 13, 1957)

IN two recent papers ${ }^{1,2}$ it was reported that when solutions of aromatic azocompounds are irradiated at temperatures low enough to prevent thermal isomerization, a photoisomerization equilibrium is established 